\title{
Composição de argilas organofílicas obtida com tensoativo não-iônico para fluidos de perfuração base orgânica
}

\section{(Compositions of organoclays obtained with nonionic surfactant for organic base drilling fluids)}

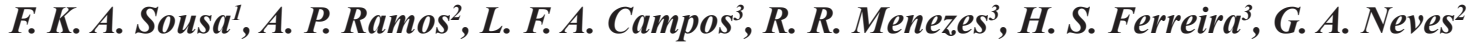 \\ ${ }^{1}$ Unidade Acadêmica de Tecnologia do Desenvolvimento, UATEC, CDSA, UFCG, R. Luiz Grande s/n, \\ Bairro Frei Damião, Sumé, PB 58540-000 \\ ${ }^{2}$ Unidade Acadêmica de Engenharia de Materiais, UAEMa, CCT, UFCG \\ ${ }^{3}$ Departamento de Engenharia de Materiais, DEMat, CT,UFPB \\ kegealves@ufcg.edu.br,gelmires@dema.ufcg.edu.br,romualdomenezes@gmail.com,liszandra@hotmail.com
}

\begin{abstract}
Resumo
Este trabalho tem como objetivo o estudo do efeito da composição de misturas de argilas bentoníticas organofílicas na reologia de fluidos de perfuração base orgânica. As composições foram estudadas por meio de delineamento de misturas, viscosidade aparente, plástica e limite de escoamento e dos testes recomendados pelo Instituto Americano de Petróleo, API (peso específico, estabilidade elétrica e volume de filtrado). Os resultados foram comparados aos obtidos com fluido padrão utilizado pela Petrobrás. Dentre as composições desenvolvidas, duas apresentaram potencial de utilização em fluidos de perfuração base orgânica, atendendo a maioria das propriedades. Foram desenvolvidas composições contendo as argilas de qualidade "inferior" (Bofe e verde-lodo) em maior quantidade e o mínimo da argila Chocolate UBM, considerada como a melhor argila da região das minas de Boa Vista, PB, para uso em fluidos de perfuração.

Palavras-chave: fluidos de perfuração, reologia, aditivo não-iônico.
\end{abstract}

Abstract

This paper aims to study the effect of compositions of bentonite organoclays for drilling fluids containing organic base. The compositions were studied using the mixture design of experiment methodology and their performance evaluated by rheological behavior (flow curves, the initial gel, final gel apparent viscosity, plastic, and flow limit) and the tests recommended by API (specific gravity, electrical stability and filtrate volume). Results were compared to those obtained with standard fluid used by Petrobrás. The results showed that among the developed compositions, two compositions showed promising potential for use in organic drilling fluids, achieving most of the limits of the normalization. Compositions containing high amount of low quality clays (Bofe and verde-lodo) and a minimum of high quality clay (Chocolate) of the mines of Boa Vista PB were developed for using in drilling fluids operation.

Keywords: drilling fluids, rheology, non-ionic additive.

\section{INTRODUÇÃOO}

As argilas organofílicas foram introduzidas nos fluidos de perfuração na década de cinqüenta do século passado, atuando como controladores da reologia do sistema [1]. Essas argilas são produzidas, em geral, a partir da reação de troca catiônica de uma argila (hidrofílica) com sais quaternários de amina ou de amônio [2]. As argilas organofílicas quando dispersa no meio oleofílico promove a capacidade de gelificação do fluido de perfuração, em virtude da característica tixotrópica da argila [3], sendo utilizadas em concentrações de 2,0 a $15 \mathrm{lb} / \mathrm{bbl}(0,0057$ a $0,0427 \mathrm{~g} / \mathrm{mL})$, dependendo da densidade do fluido formulado [1].

Os fluidos de perfuração consistem em uma fase líquida contínua, tratada quimicamente de forma a adequar suas propriedades às operações de perfuração [4-6]. Suas propriedades principais são massa específica (na prática de fluidos de perfuração, há certa imprecisão na nomenclatura, como por exemplo: os termos densidade, massa específica e peso específico. Que serão utilizados indistintamente neste texto com o significado de massa específica), viscosidade, homogeneidade, reatividade, filtrado e salinidade. Os fluidos de perfuração em relação ao meio dispersante líquido podem ser de dois tipos: fluidos base de água e fluidos base orgânica. Os fluidos base orgânica são formados por uma emulsão na qual a fase contínua é um liquido não aquoso e água saturada com $\mathrm{NaCl}$, tendo como fase sólida argilas organofílicas.

Dentre o grande número de composições de argilas organofílicas (argila mais componente orgânico) pesquisadas, as de maior importância industrial são as 
organofílicas obtidas a partir de argilas bentoníticas sódicas (com alto grau de inchamento em água) e sais quaternários de amônio [7].

Atualmente a Petrobrás utiliza argilas organofilicas importadas na formulação dos fluidos base orgânica, verificando-se uma enorme necessidade de desenvolvimento de produtores nacionais de argilas organofílicas para tal aplicação, visando à substituição de produtos importados por produtos nacionais $[4,8]$. Até a década de 60 , o Brasil importava toda a bentonita utilizada em fluidos de perfuração. Hoje, as jazidas de Boa Vista, PB, tornam o país praticamente auto-suficiente em argilas bentoníticas, sendo responsáveis por $88 \%$ de toda a produção de bentonita bruta beneficiada do Brasil $[5,6]$. Nos primeiros anos de exploração, era possível encontrar argilas de várias tonalidades, com diferentes capacidades de expansão em água e formação de géis tixotrópicos, sendo muitas delas consideradas de excelente qualidade para uso em fluidos de perfuração. No entanto, atualmente, muitas das variedades encontram-se esgotadas e outras começando a rarear, a exemplo da argila denominada localmente por Chocolate, considerada como de boa qualidade para uso em fluidos de perfuração. Há uma grande quantidade de outras argilas, como as denominadas por Bofe e Verde-lodo, no entanto, essas argilas apresentam propriedades reológicas que limitam seu uso em fluidos de perfuração [5].

O desaparecimento das variedades de argilas de melhor qualidade e o crescente consumo de argilas para aplicação em fluidos de perfuração vem sendo uma grande motivação para o desenvolvimento de pesquisas [9-11] visando aumentar o uso das argilas de "má qualidade" para fluidos de perfuração e otimizar a utilização das que apresentam melhores características reológicas. Resultados obtidos em pesquisas realizadas anteriormente $[9,10]$ visando otimizar composições de argilas (misturas de argilas) para fluidos base água evidenciaram que a adequada formulação de composições possibilita aumentar a quantidade de argilas ditas de "má qualidade", otimizando o uso das mais nobres em fluidos de perfuração base água, ao mesmo tempo que se obtém fluidos com propriedades reológicas que atendem os requisitos para uso em fluidos de perfuração para poços de petróleo. Ainda nesse sentido, estudos [11], também utilizando as argilas de Boa Vista, PB, evidenciaram que a purificação das argilas permite obter argilas organofílicas com maior inchamento em óleo, mesmo quando da utilização das argilas de "má qualidade"para uso em fluidos de perfuração.

No entanto, a purificação pode encarecer o custo da matéria-prima e ampliar significativamente o tempo de processamento do material. Diante do exposto, observa-se a necessidade da ampliação dos estudos de síntese de argilas organofílicas a partir das bentonitas de Boa Vista, visando utilizar e otimizar o uso das argilas de menor qualidade, disponíveis em maior quantidade nas jazidas (argilas Bofe e verde-lodo) para utilização em fluidos de perfuração base orgânica. Assim, esse trabalho tem por objetivo o estudo do efeito da composição de misturas de argilas bentoníticas organofílicas na reologia de fluidos de perfuração base orgânica.

\section{MATERIAIS E MÉTODOS}

Foram utilizadas três amostras de argilas bentoníticas policatiônicas proveniente da Mina Nova, argila Chocolate UBM, e da Mina Bravo, argilas verde-lodo e Bofe. Foi utilizada também uma argila organofílica padrão utilizada pela Petrobrás, para fins de comparação dos resultados, cedida pela Petrobrás. Os resultados de caracterização física, química e mineralógica já foram reportados [12]. Foi utilizado um tensoativo não-iônico de amina etoxilada, (Oxiteno Ind. Com. Ltda.) As argilas foram organofilizadas individualmente, com um teor de $30 \%$ em massa de tensoativo não iônico. $\mathrm{O}$ detalhamento do processo de organofilização já foi reportado [12]. As argilas organofilizadas foram caracterizadas por difração de raios X (Shimadzu, XRD 6000) e análise termogravimétrica (Shimadzu, TA 60h).

A análise das composições de argilas (mistura de argilas) para serem usadas como argilas organofílicas em fluidos de perfuração foi realizada com planejamento experimental por delineamento de mistura, com um planejamento em rede simplex $\{3,2\}$ com ponto central aumentado. A Tabela I apresenta as composições formuladas e usadas para avaliação das propriedades reológicas dos fluidos.

Tabela I - Composições obtidas através do planejamento em rede simplex centróide $\{3,2\}$, aumentado.

[Table I - Compositions obtained by planning an increased network simplex centroid \{3.2\}.]

\begin{tabular}{cccc}
\hline \multicolumn{4}{c}{ Proporções das argilas organofílicas (\%) } \\
\hline Composição & Bofe & Chocolate & Verde-lodo \\
\hline 1 & 100,0 & 0,0 & 0,0 \\
2 & 0,0 & 100,0 & 0,0 \\
3 & 0,0 & 0,0 & 100,0 \\
4 & 50,0 & 50,0 & 0,0 \\
5 & 50,0 & 0,0 & 50,0 \\
6 & 0,0 & 50,0 & 50,0 \\
7 & 33,3 & 33,3 & 33,3 \\
8 & 66,6 & 16,7 & 16,7 \\
9 & 16,7 & 66,6 & 16,7 \\
10 & 16,7 & 16,7 & 66,6 \\
\hline
\end{tabular}

Para a preparação dos fluidos de perfuração, foram utilizados os seguintes aditivos: emulsificantes, salmoura, ativador, redutor de filtrado, viscosificante e adensante (aditivos utilizados normalmente para a preparação de fluidos base orgânica). Os aditivos estão descritos na Tabela II. A mistura de componentes foi feita com o auxilio de um agitador Hamilton Beach sob rotação de 17000 rpm. Primeiramente foram adicionados os emulsificantes juntamente com o éster (fase contínua), agitou-se a mistura por $20 \mathrm{~min}$, adicionou-se a salmoura [13] com posterior agitação por mais $10 \mathrm{~min}$. Após, adicionou-se o ativador, 
e agitou-se por $10 \mathrm{~min}$. Nesse momento, adicionou-se o modificador reológico (argila organofílica) e manteve-se a agitação por 10 min. Em seguida, adicionou-se o redutor de filtrado e agitou-se por $10 \mathrm{~min}$. Por fim, adicionou-se o adensante ao fluido, e agitou-se a mistura por $10 \mathrm{~min}$. Ao final desse processo o peso específico do fluido foi medido em uma balança densimétrica. Se o valor medido fosse menor que o desejado, seria adicionado mais adensante. $\mathrm{O}$ fluido foi então transferido para uma célula e envelhecido em estufa rotativa (roller-over) durante $16 \mathrm{~h}$ a $66^{\circ} \mathrm{C}$. O objetivo do envelhecimento de um fluido de perfuração é simular a utilização do fluido durante uma perfuração, ou seja, a sua circulação através de todo o sistema de perfuração. Ao final desse processo, foram feitos os testes de estabilidade elétrica e reologia dos fluidos.

Tabela II - Aditivos usados nos fluidos base orgânica. [Table II - Additives used in organic base fluids.]

\begin{tabular}{cc}
\hline Componentes do fluido & Quantidade \\
\hline Emulsificantes & Variável (entre 6 e 12 lb/bbl) \\
Éster (fase contínua) & Variável $(50-80 \%$ ) \\
Salmoura (fase aquosa) & Variável $(20-50 \%)$ \\
Ativador & $4,0 \mathrm{~g} / 350 \mathrm{~mL}$ fluido \\
Redutor de filtrado & $2,0 \mathrm{~g} / 350 \mathrm{~mL}$ fluido \\
Argila organofílica & $2,0 \mathrm{~g} / 350 \mathrm{~mL}$ fluido \\
(viscosificante) & $8,0-8,5 \mathrm{lb} / \mathrm{gal}$ \\
Adensante & 1
\end{tabular}

Após a obtenção do fluido formulado foram efetuadas as medidas de viscosidade com viscosímetro Fann 35A para medir a viscosidade aparente (VA) e plástica (VP), o limite de escoamento (LE), medidas de gel inicial (GI) e gel final (GF) dos fluidos de perfuração [13, 14]. O objetivo dos testes de gel final e gel inicial é avaliar a capacidade que o fluido tem em reter os detritos, quando a perfuração é interrompida. A diferença na metodologia de análise do gel inicial e do gel final está no tempo de repouso. Para o gel inicial o repouso antes de se iniciar o cisalhamento é de $10 \mathrm{~s}$, já para o gel final é de 10 min. Dessa maneira é possível verificar se a atuação do modificador reológico é rápida ou lenta, quando se inicia a perfuração após uma pausa. A faixa estipulada pelo API [14] para o gel inicial é $1,44-5,75 \times 10^{-6} \mathrm{MPa}\left(3-12 \mathrm{lbf} / 100 \mathrm{ft}^{2}\right)$

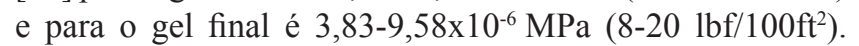
Valores de gel muito elevados são indesejáveis, pois ao retornar a perfuração após uma parada, a alta viscosidade exigiria grande esforço no sistema de bombas de fluido. Já valores de gel muito abaixo podem provocar entupimento do poço devido a não sustentação dos detritos pelo gel. Em seguida foram determinados o peso específico (PE) do fluido em uma balança densimétrica e a estabilidade elétrica (PE) [14] O valor de estabilidade elétrica (EE) indica o quanto à emulsão formada é estável. Através do teste é possível verificar se ocorre a separação das fases aquosa e de óleo do fluido. O ideal é que não ocorra esta separação, pois o fluido tem que ser estável para manter em suspensão os detritos gerados durante a perfuração. O valor ótimo estipulado pelo API [14] é $300 \mathrm{~V}$. A estabilidade elétrica do fluido foi medida com um aparelho de estabilidade elétrica Fann 23D. A determinação do volume de filtrado (VF) dos fluidos foi realizada com um filtro prensa HTHP Fann com temperatura do termostato mantida em $94{ }^{\circ} \mathrm{C}$, regulando-se a pressão para $0,7 \mathrm{MPa}$ (100 psi), coletou-se o volume de filtrado após $30 \mathrm{~min}$. O valor estipulado pelo API é de no máximo $6 \mathrm{~mL}$ de volume de filtrado. A Tabela III apresenta as propriedades avaliadas e os valores ótimos segundo o API.

Tabela III - Propriedades avaliadas e valores ótimos segundo o API [14].

[Table III - Properties assessed and optimum values according to the API [14].]

\begin{tabular}{ll}
\hline Propriedades & Valor API \\
\hline Peso específico (lb/gal) & $8,0-8,5$ \\
Estabilidade elétrica $(\mathrm{V})$ & $\sim 300$ \\
Viscosidade plástica $(\mathrm{cP})$ & 25 a 60 \\
Gel inicial $\left(\mathrm{lbf} / 100 \mathrm{ft}^{2}\right)$ & 3 a 12 \\
Gel final $\left(\mathrm{lbf} / 100 \mathrm{ft}^{2}\right)$ & 8 a 20 \\
Limite de escoamento $\left(\mathrm{lbf} / 100 \mathrm{ft}^{2}\right)$ & 5 a 20 \\
Volume de filtrado $(\mathrm{mL})$ & $<6$ \\
\hline
\end{tabular}

\section{RESULTADOS E DISCUSSÃO}

As Figs. 1, 2 e 3 apresentam os difratogramas de raios $\mathrm{X}$ das argilas organofilizadas obtidas com as argilas Bofe, Chocolate UBM e verde-lodo tratadas com o tensoativo não iônico no teor de $30 \%$ e da argila padrão. A argila Bofe não modificada apresentou espaçamento basal $\mathrm{d}_{001}$ de 1,48 nm e espaçamentos máximos correspondendo às distâncias oriundas de intercalação, de 1,71 nm e 3,41 nm quando tratada com tensoativo não iônico. A argila Chocolate UBM não modificada apresentou espaçamento basal $d_{001}$ de 1,55 nm e espaçamentos máximos correspondendo

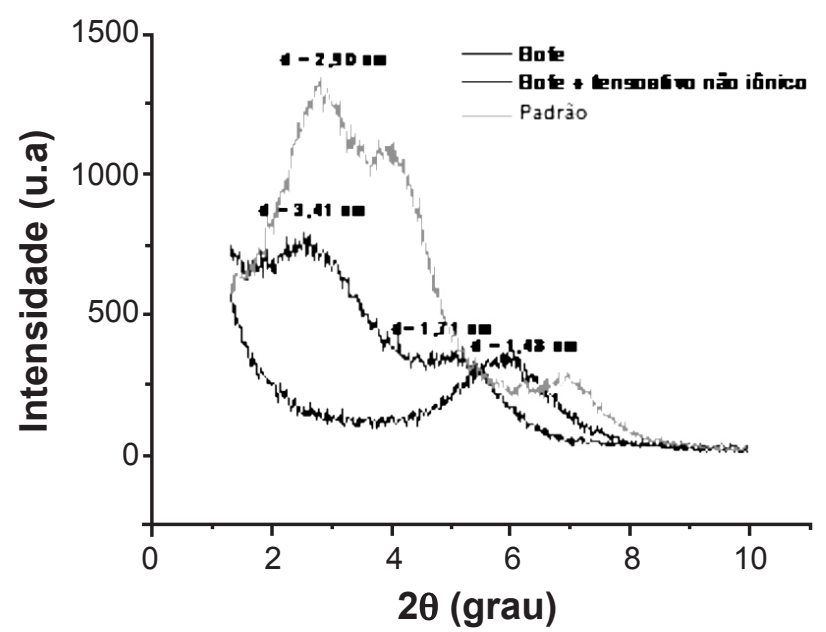

Figura 1: Difratogramas de raios $\mathrm{X}$ da argila Bofe modificada com tensoativo não-iônico.

[Figure 1: X-ray diffraction patterns of Bofe clay modified with non-ionic surfactant.] 


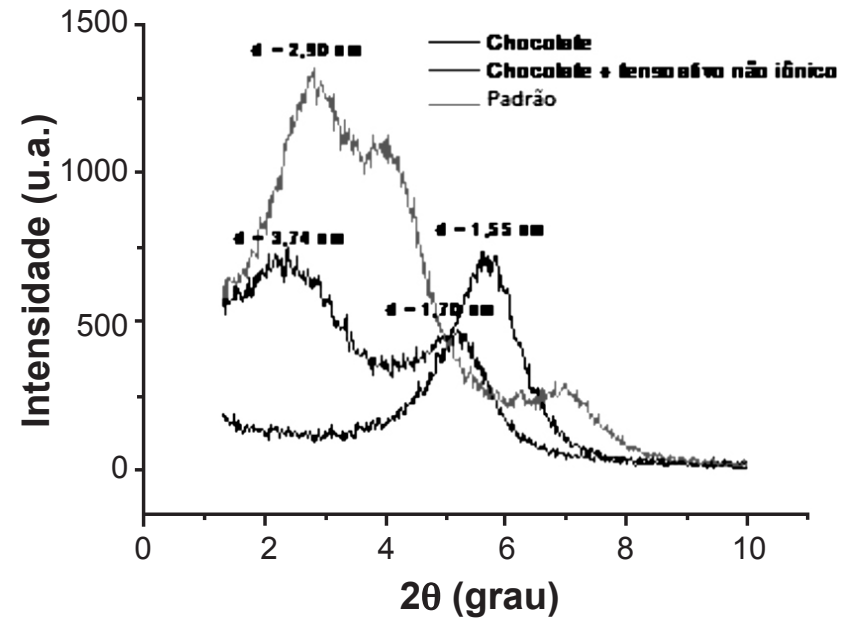

Figura 2: Difratogramas de raios $\mathrm{X}$ da argila Chocolate UBM modificada com o tensoativo não-iônico.

[Figure 2: X-ray diffraction patterns of clay Chocolate UBM modified with non-ionic surfactant.]

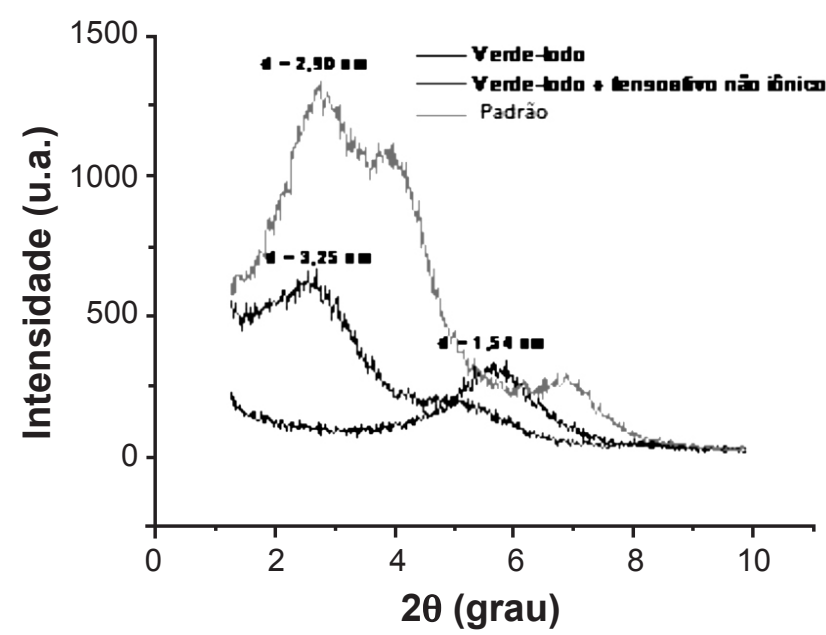

Figura 3: Difratogramas de raios X da argila Verde-lodo modificada com o tensoativo não-iônico.

[Figure 3: X-ray diffraction patterns of Clay Verde-lodo modified with non-ionic surfactant.]

às distâncias oriundas de intercalação de $1,70 \mathrm{~nm}$ e 3,74 nm após organofilização. A argila verde-lodo não modificada apresentou espaçamento basal $\mathrm{d}_{001}$ de $1,54 \mathrm{~nm}$ e espaçamentos máximos correspondendo às distâncias oriundas de intercalação de 3,25 nm, quando organofilizada.

A presença de dois valores de espaçamentos de intercalação nos dois tipos de argilas (Bofe e Chocolate UBM) indica que algumas camadas são mais facilmente intercaladas com o sal e que outras, por outro lado, são mais difíceis de serem intercaladas gerando, assim, espaçamentos basais diferentes [15]. O aparecimento de vários espaçamentos basais durante a intercalação do tensoativo não iônicos parece estar associado à distribuição heterogênea de cargas na camada [16-18]. Observando-se os difratogramas da argila padrão é possível verificar os espaçamentos basais de 2,90 nm e 2,12 nm, que confirmam a intercalação do sal nessa argila organofílica. O pico característico do argilomineral esmectítico com espaçamento basal de 1,25 nm indica a não penetração do sal quaternário de amônio, entre algumas camadas da argila.

Na Fig. 4 estão apresentadas as curvas de fluxo das composições 1, 2 e 3, organofilizadas com amina etoxilada e da argila padrão. A Fig. 5 mostra as curvas de fluxo das composições 4, 5, 6 e da argila padrão e a Fig. 6 contem as curvas de fluxo das composições 7, 8, 9, 10 e da argila padrão.

Analisando-se as Figs. 4, 5 e 6, observa-se que os fluidos das composições desenvolvidas apresentaram comportamento semelhante, porém sempre com valores de viscosidade inferiores ao fluido da argila padrão. Verificase também, que as curvas de fluxo indicam que os fluidos desenvolvidos apresentam comportamento pseudoplástico. Este tipo de comportamento pode ser causado por diversos fatores, dentre os quais as características físicas das

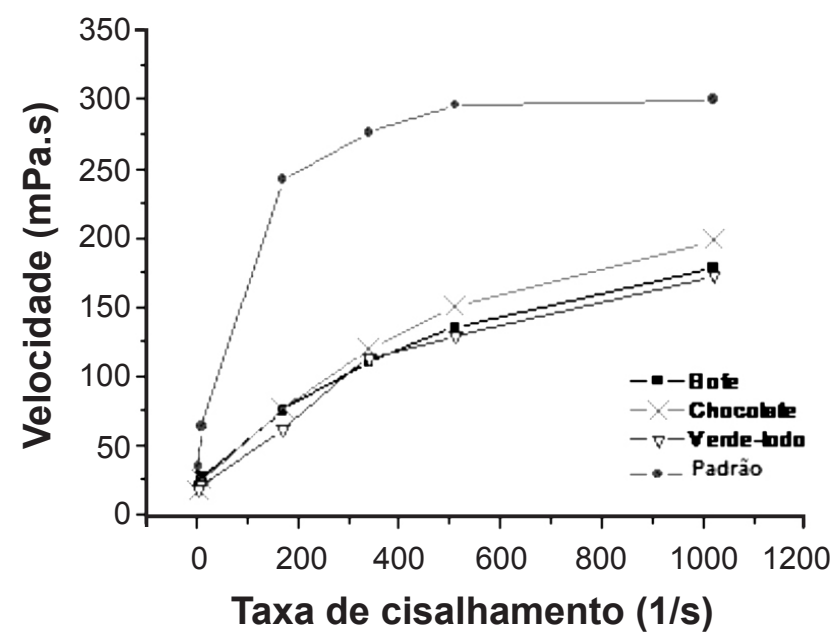

Figura 4: Curva de fluxo dos fluidos formulados com as composições 1, 2 e 3, organofilizadas e da argila padrão.

[Figure 4: Flow curve of fluids formulated with the compositions 1, 2 and 3 and standard clay.]

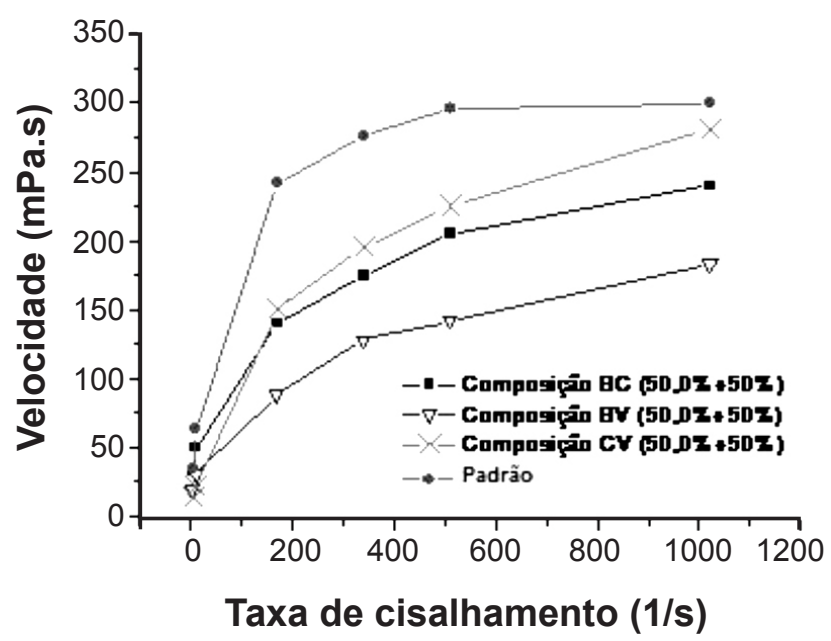

Figura 5: Curva de fluxo dos fluidos formulados com as composições 4, 5, 6 e da argila padrão.

[Figure 5: Flow Curve of fluids formulated with the compositions 4, 5, 6 and standard clay.] 


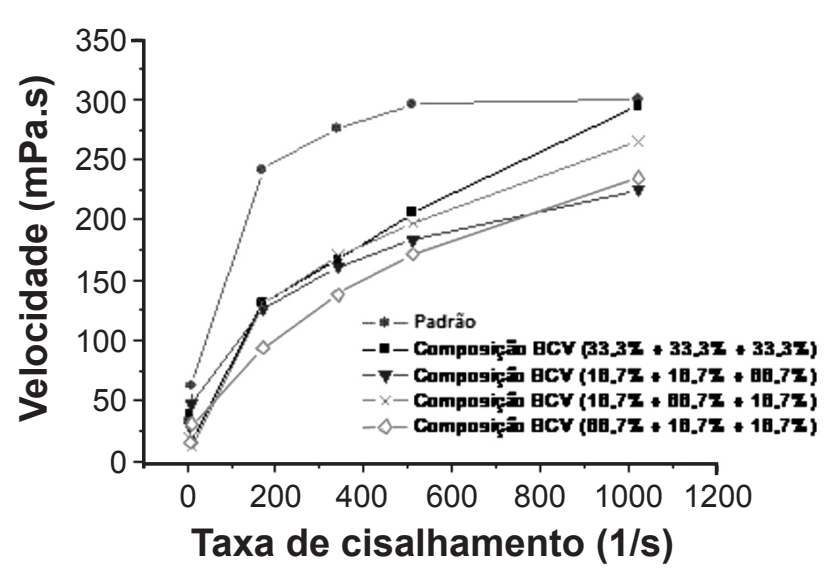

Figura 6: Curva de fluxo dos fluidos formulados com as composições 7, 8, 9, 10 e da argila padrão.

[Figure 6: Flow curve of fluids formulated with the compositions 7, 8, 9, 10 and standard clay.]

partículas, como sua área superficial, formato e dimensões; o tipo de interação entre as partículas (atração ou repulsão), e a concentração, peso molecular e conformação de moléculas de dispersantes presentes no meio liquido. A presença de aglomerados relativamente fracos na suspensão, originados pela atuação de forças de interação atrativas entre partículas, pode ser considerada a principal causa do fenômeno de pseudoplasticidade.

A Tabela IV apresenta os resultados de gel inicial (GI), gel final (GF), viscosidade aparente (VA) e limite de escoamento (LE) para os fluidos formulados com as composições de argilas organofílicas e com a argila padrão. Analisando os dados de gel inicial para os fluidos, observase que todos os valores ultrapassaram o limite máximo estipulado pelo API [14], sendo os melhores resultados apresentados pelos fluidos 7,8 e 9. Analisando os dados de gel final para os fluidos, observa-se que a maioria dos valores atende a faixa de aceitação estipulada pelo API [14], sendo os melhores resultados apresentados pelos fluidos 2 ,
3, 5, 7, 8 e 9, enquanto que o fluido padrão apresentou valor fora da faixa indicada pelo API [14].

A viscosidade aparente dos fluidos formulados com a argila padrão encontra-se próxima e pouco superior a de todos os fluidos formulados com as composições de argilas bentoníticas organofilizadas, o que já foi evidenciado nas curvas de fluxos dos fluidos. Os fluidos apresentaram elevados valores de viscosidade plástica, com a maioria (fluidos 1, 2, 3, 4, 5, 6 e 10) apresentando valores que atendem a faixa recomendada pela API [14]. O fluido formulado com a argila padrão apresentou valor inferior ao mínimo estipulado pelo API [14]. Analisandose os resultados obtidos para o limite de escoamento, observase que os fluidos apresentam limite de escoamento superior ao indicado pelo API [14] em todos os casos.

A Tabela $\mathrm{V}$ apresenta os resultados de peso específico, estabilidade elétrica e volume de filtrado para os fluidos formulados. Em todos os fluidos analisados, o peso específico foi de $0,98 \mathrm{~g} / \mathrm{cm}^{3}$ (8,2 lb/gal), dentro da faixa indicada. Os fluidos apresentaram valores de estabilidade elétrica que atendem ao estipulado pelo API [14]. Por outro lado, observa-se que apenas os fluidos 7, 8, 9, 10 e o padrão apresentaram valores de volume de filtrado que atendem as especificações da API [14].

A partir dos resultados obtidos e utilizando-se uma replicata foram geradas equações de regressão visando correlacionar as proporções das argilas nas composições com os parâmetros GI, GF, VA, VP, LE e VF. Foram escolhidas as equações que apresentaram significância estatística ao nível de $95 \%$ de confiança e os maiores coeficientes de correlação. Os modelos matemáticos obtidos correlacionando os valores de GI, GF, VA, VP, LE e VF com as proporções das argilas organofílicas Bofe, Chocolate UBM e Verde-lodo são apresentados na Tabela VI. Onde os termos B, C e V representam as frações mássicas das argilas Bofe, Chocolate e Verde-lodo respectivamente.

Todos os coeficientes são estatisticamente significativos ao nível de $95 \%$ de confiança. Os componentes da mistura

Tabela IV - Comportamento reológico das composições no meio dispersante éster.

[Table IV - Rheological behavior of compositions in ester.]

\begin{tabular}{cccccc}
\hline Composição & $\begin{array}{c}\text { GI } \\
\left(\mathrm{lbf} / 100 \mathrm{ft}^{2}\right)\end{array}$ & $\begin{array}{c}\text { GF } \\
\left(\mathrm{lbf} / 100 \mathrm{ft}^{2}\right)\end{array}$ & $\mathrm{VA}(\mathrm{cP})$ & $\mathrm{VP}(\mathrm{cP})$ & $\mathrm{LE}\left(\mathrm{lbf} / 100 \mathrm{ft}^{2}\right)$ \\
\hline 1 & 20,0 & 21,0 & 89,0 & 43,0 & 46,0 \\
2 & 17,0 & 15,5 & 99,0 & 48,0 & 51,0 \\
3 & 17,5 & 16,0 & 86,0 & 43,5 & 42,5 \\
4 & 32,0 & 38,0 & 120,0 & 35,0 & 85,0 \\
5 & 19,0 & 18,0 & 91,5 & 41,0 & 50,5 \\
6 & 35,0 & 29,0 & 140,5 & 56,0 & 84,5 \\
7 & 13,5 & 13,5 & 147,3 & 88,5 & 58,8 \\
8 & 13,0 & 12,0 & 117,5 & 63,0 & 54,5 \\
9 & 15,0 & 13,5 & 132,5 & 68,0 & 64,5 \\
10 & 26,0 & 21,0 & 112,5 & 42,0 & 70,5 \\
Padrão & 31,0 & 27,0 & 150,0 & 4,0 & 146,0 \\
\hline
\end{tabular}


Tabela V - Testes recomendados pelo API [14]. [Table V - Recommended tests by API [14].]

\begin{tabular}{cccc}
\hline Composição & PE (lb/gal) & EE (V) & VF (mL) \\
\hline 1 & 8,2 & 380,0 & 14,2 \\
2 & 8,2 & 380,0 & 14,4 \\
3 & 8,2 & 380,0 & 12,2 \\
4 & 8,2 & 382,0 & 17,0 \\
5 & 8,2 & 382,0 & 14,6 \\
6 & 8,2 & 382,0 & 6,2 \\
7 & 8,2 & 380,0 & 2,4 \\
8 & 8,2 & 375,0 & 3,0 \\
9 & 8,2 & 375,0 & 2,6 \\
10 & 8,2 & 375,0 & 2,8 \\
Padrão & 8,2 & 286,0 & 3,2 \\
\hline
\end{tabular}

interagem sinergicamente quando os coeficientes das interações são maiores que zero contribuindo para elevar os valores das propriedades e interagem antagonisticamente quando os coeficientes das interações contribuem para diminuir seus valores [6]. Analisando os termos estatisticamente significativos dos modelos ajustados para GI, GF, VA e LE, as argilas Bofe e Chocolate UBM interagem sinergicamente contribuindo para aumentar os valores dessas propriedades, o mesmo acontece para interação entre as argilas Chocolate UBM e verde-lodo. As argilas Bofe, Chocolate UBM e verde-lodo interagem antagonisticamente, isto é, contribuem para diminuir GI, GF e LE e contribuem para aumentar VA. Analisando os coeficientes do modelo ajustado para a VP, observa-se que as argilas Bofe, Chocolate UBM e verde-lodo interagem sinergicamente para aumentar VP. Observando os coeficientes do modelo obtido para o VF, verifica-se que as argilas Bofe e Chocolate UBM interagem sinergicamente contribuindo para aumentar VF, o mesmo acontece para interação entre Bofe e verde-lodo e entre Chocolate UBM e verde-lodo. As argilas Bofe, Chocolate UBM e verde-lodo interagem antagonisticamente, isto é, contribuem para diminuir VF.

A Tabela VII apresenta os parâmetros estatísticos para os modelos ajustados e apresentados na Tabela VI. Analisando os parâmetros estatísticos principais (Teste F, Valor $\mathrm{p}$, coeficiente de múltipla determinação, $\mathrm{R}^{2}$ e o valor da razão entre o teste $\mathrm{F}$ calculado e o teste $\mathrm{F}$ tabelado no nível de $95 \%$ de confiança), observa-se que os modelos são estatisticamente significativos ao nível estipulado (valor $\mathrm{p} \leq$ nível de significância), que $\mathrm{R}^{2}$ mostra que os modelos ajustados para GI, GF, VA, VP, LE e VF, para todas as condições de aditivação, não apresentam variabilidades consideráveis; quanto mais perto da unidade estiver o valor de $\mathrm{R}^{2}$, melhor terá sido o ajuste do modelo aos dados observados.

Para que uma regressão não seja apenas estatisticamente significativa, mas também útil para realizar previsões, o valor da razão entre o $\mathrm{F}$ calculado e o $\mathrm{F}$ tabelado deve ser no

Tabela VII - Estatísticas relevantes para análise de variância das variáveis GI, GF, VA, VP, LE e VF, todas ajustadas ao Modelo Cúbico Especial.

[Table VII - Relevant statistics of the analysis of variance of GI, GF, VA, VP, LE and VF, all adjusted to the Special Cubic Model.]

\begin{tabular}{ccccr}
\hline Variáveis & Teste $\mathrm{F}_{\text {calc }}$ & Valor $\mathrm{p}$ & $\mathrm{R}^{2}$ & $\mathrm{~F}_{\text {calc }} / \mathrm{F}_{\text {tab }}$ \\
\hline $\mathrm{GI}$ & 38,4541 & 0,0000 & 0,7881 & 11,0500 \\
$\mathrm{GF}$ & 40,4357 & 0,0000 & 0,7897 & 11,6194 \\
$\mathrm{VA}$ & 29,4268 & 0,0000 & 0,9686 & 8,4560 \\
$\mathrm{VP}$ & 39,9790 & 0,0000 & 0,7257 & 11,4882 \\
$\mathrm{LE}$ & 19,1774 & 0,0006 & 0,8838 & 5,5107 \\
$\mathrm{VF}$ & 45,9084 & 0,0000 & 0,8283 & 13,1921 \\
\hline
\end{tabular}

Tabela VIII - Resultados dos testes de falta de ajuste para as variáveis estudadas (Modelo Cúbico Especial).

[Table VIII - Results of lack adjustment tests for studied variables (Special Cubic Model).]

\begin{tabular}{ccc}
\hline Variáveis & Teste F para falta de ajuste & Valor $\mathrm{p}$ \\
\hline $\mathrm{GI}$ & 10,4136 & 0,0002 \\
$\mathrm{GF}$ & 10,5144 & 0,0002 \\
$\mathrm{VA}$ & 86,3239 & 0,0000 \\
$\mathrm{VP}$ & 14,1110 & 0,0000 \\
$\mathrm{LE}$ & 21,3040 & 0,0000 \\
$\mathrm{VF}$ & 10,4573 & 0,0002 \\
\hline
\end{tabular}

Tabela VI - Equações correlacionando os valores de GI, GF, VA, VP, LE e VF com as frações mássicas das argilas organofílicas Bofe, Chocolate UBM e verde-lodo. [Table VI - Equations correlating the values of GI, GF,VA,VP, LE and VF with mass fraction of organoclays Bofe, Chocolate UBM and verde-lodo.]

\begin{tabular}{l}
\hline $\mathrm{GI}=19,25 \mathrm{~B}+15,27 \mathrm{C}+20,25 \mathrm{~V}+47,80 \mathrm{BC}+71,80 \mathrm{CV}-515,22 \mathrm{BCV}$ \\
\hline $\mathrm{GF}=19,41 \mathrm{~B}+13,88 \mathrm{C}+18,14 \mathrm{~V}+72,81 \mathrm{BC}+54,26 \mathrm{CV}-557,15 \mathrm{BCV}$ \\
\hline $\mathrm{VA}=90,94 \mathrm{~B}+98,45 \mathrm{C}+84,99 \mathrm{~V}+102,32 \mathrm{BC}+184,41 \mathrm{CV}+496,04 \mathrm{BCV}$ \\
\hline $\mathrm{VP}=39,80 \mathrm{~B}+49,80 \mathrm{C}+40,13 \mathrm{~V}+1032,00 \mathrm{BCV}$ \\
\hline $\mathrm{LE}=47,46 \mathrm{~B}+48,89 \mathrm{C}+48,68 \mathrm{~V}+134,63 \mathrm{BC}+149,08 \mathrm{CV}-529,91 \mathrm{BCV}$ \\
\hline $\mathrm{VF}=2,89 \mathrm{~B}+2,79 \mathrm{C}+2,49 \mathrm{~V}+2,10 \mathrm{BC}+1,33 \mathrm{BV}+2,10 \mathrm{CV}-23,55 \mathrm{BCV}$ \\
\hline
\end{tabular}


mínimo de quatro a cinco vezes o valor do F tabelado [20]. Essa condição é amplamente satisfeita para todos os modelos encontrados. O teste para falta de ajuste está apresentado na Tabela VIII. Analisando os dados observa-se que os modelos ajustados para GI, GF, VA, VP, LE e VF (Tabela VI) de uma maneira geral são aceitáveis. Observa-se também que GI, GF, VA, VP, LE e VF apresentaram falta de ajuste (Valor $\mathrm{p}$ $<0,05)$ justificada, provavelmente, pelo fato de que algumas dispersões apresentaram-se no estado floculado-gel, estado em que ocorre grande variação dos parâmetros reológicos, contribuindo para uma variabilidade nas replicatas obtidas.

Os modelos ajustados podem ser tomados como sendo estatisticamente significativos para descrever $\mathrm{o}$ comportamento de GI, GF, VA, VP, LE e VF em função das proporções das argilas na composição.

\section{CONCLUSÃO}

Os fluidos desenvolvidos com as composições de argilas apresentaram comportamento pseudoplástico; composições de argilas contendo argilas de "má qualidade" possibilitam obter fluidos que apresentaram resultados reológicos que atendem a maioria das recomendações da normatização; a interação entre as argilas Bofe e Chocolate UBM contribui para aumentar os valores de GI, GF, VA, LE e VF, o mesmo acontece para interação entre as argilas Chocolate UBM e verde-lodo. A interação entre as argilas Bofe, Chocolate UBM e verde-lodo contribui para diminuir GI, GF e LE e contribuem para aumentar VA e VP. Os fluidos desenvolvidos apresentam comportamento reológico melhor que o observado utilizando a argila padrão organofilizada, o que indica que as composições desenvolvidas apresentam potencial tecnológico para aplicação em perfuração de poços de petróleo.

\section{AGRADIMENTOS}

Ao CNPq pela concessão da bolsa de estudo, à Oxiteno Ind. e Com. Ltda. pelo fornecimento dos aditivos e ao Laboratório de Reciclagem da UAEMa, CCT, UFCG, pelo uso de suas instalações físicas.

\section{REFERÊNCIAS}

[1] H. C. Darley, G. R. Gray, Composition and properties of drilling and completion fluids, 5a Ed., Gulf Publ. Co., Houston, Texas, EUA (1988) p. 625.

[2] H. S. Ferreira, Obtenção de argilas organofilicas purificadas para uso em fluidos de perfuração base orgânica, Diss. Mestrado, Coordenação de Pós Graduação em Ciência e Engenharia de Materiais, UFCG, Campina
Grande, PB (2005).

[3] R. Caenn, G. V. Chillingarm, J. Petroleum Sci. Eng. 14 (1996) 221-230.

[4] L. V. Amorim, E. Pereira, Bentonita da Paraiba: A exaustão de uma riqueza brasileira, ABAS Informa 147 (2003).

[5] L. F. A. Campos, Composições de argilas bentoníticas para utilização em fluidos de perfuração de poços de petróleo, Tese Dr., Coordenação de Pós-Graduação em Engenharia de Processos, UFCG, Campina Grande, PB (2007).

[6] P. S. Santos, Ciência e Tecnologia de Argilas, $3^{\mathrm{a}}$ Ed., Vol. 1. Edgard Blücher, S. Paulo, SP (1992) p. 408.

[7] F. R. Valenzuela Diaz, Preparação a nível de laboratório de algumas argilas esmectiticas organofilicas, Tese Dr., Departamento de Engenharia Química, Escola Politécnica da Universidade de S. Paulo, SP (1994).

[8] M. L. Oliveira, Bentonita, www.dnpm.org.br, 12/2004, acesso em 12/12/2006.

[9] L. F. A. Campos, L. V. Amorim , H. C. Ferreira, Cerâmica 52 (2006) 69-75.

[10] L. F. A. Campos, L. V. Amorim , H. C. Ferreira, Cerâmica 53 (2007) 68-73

[11] H. S. Ferreira, R. R. Menezes, H. S. Ferreira, A. B. Martins, G. A. Neves, H. C. Ferreira, Cerâmica 54 (2008) 77-85

[12] H. S. Ferreira, Otimização do processo de organofilização de bentonitas visando seu uso em fluidos de perfuração não aquosas, Tese Dr., Coordenação de Pós Graduação em Engenharia de Processos, UFCG, Campina Grande, PB (2009).

[13] Petrobras, Ensaio de argila organofilica para fluidos de perfuração à base de óleo, N-2259 (1997).

[14] American Petroleum Institute - API, Standard procedure for testing drilling fluids, API Recommended practice for field testing of oil-based drilling fuids 13B-2, Dallas, $4^{\text {th }}$ Ed. (2005).

[15] Q. Zhou, R. L. Frost, Y. He, J. Xi, Colloid Interface Sci. 307 (2007) 50-55.

[16] G. Lagaly, Solid State Ionics 22 (1984) 43-51.

[17] I. Dékány, F. Szanta, L. G. Nag, Colloid Polymer Sci. 266 (1988) 82-86.

[18] S. Y. Lee, S. J. Kim, Colloids and Surfaces A: Physicochem. Eng. Aspects 211 (2002) 19-26.

[19] J. A. Cornell, Experiments with mixtures: Designs, models and the analysis of mixture data, $3^{\mathrm{a}} \mathrm{Ed}$., John Wiley \& Sons, New York, EUA (1990) p.789.

[20] B. Barros Neto, J. S. Scarminio, R. E. Bruns, Planejamento e Otimização de Experimentos, $2^{\mathrm{a}}$ Ed., Ed. Unicamp, Campinas, SP (1996) p. 152.

(Rec. 08/04/2010, Ac. 14/07/2010) 